\title{
175. On Completely Continuous Operators on Locally Convex Vector Spaces
}

\author{
By Taira SHIROTA \\ Department of Mathematics, Osaka University \\ (Comm. by K. KunugI, M.J.A., Nov. 12, 1954)
}

The classical theorem of Riesz and Schauder concerning completely continuous operators on Banach spaces was extended on locally convex vector spaces by J. Leray. ${ }^{12}$ However the proof of the alternative of Fredholm was based on a theorem on the invariance of the domain and the theorems of Schauder for the conjugate space were incomplete.

In the present note we prove the alternative of Fredholm and the Riesz-Schauder theory in the case of locally convex vector spaces. Furthermore we concern ourselves with iterations of continuous operators and with results of M. Altman. ${ }^{2}$ The method is linear space-theoretic and is based on the Leray's results.

1. The alternative of Fredholm. Let $X$ be a locally convex vector space with complex coefficients and let $t$ be a completely continuous operator on $X$ into itself, i.e., there is a neighbourhood $U$ of 0 in $X$ such that $t(U)$ is relatively compact (compact=bicompact). Furthermore let $X^{*}$ be the space of all continuous functionals on $X$ with the compact open topology. Then we have the following

Lemma 1. The adjoint $t^{*}$ of $t$ is completely continuous with respect to the above topology on $X$.

For $t^{*}\left((t(U))^{0}\right) \subset U^{0}$ where $U^{0}=\{f|f(U)| \leqq 1\}$ and $U^{0}$ is compact in our topology by the theorem of Ascoli.

Lemma 2. Let $t$ be a completely continuous operator without eigenvalue 1. Then $(t-1)(X)=X$.

For let $v=t-1$ and suppose, on the contrary, that $v(X) \varsubsetneqq X$. Then by Lemma $1, t^{*}$ is completely continuous and $v^{*^{-1}}(0)=(v(X))^{0}$, hence by $[\mathrm{L}]$, Lemma 9.2, $v^{*^{-p}}(0)=v^{*^{-p-1}}(0) \neq\{0\}$ for some integer $\rho$. Accordingly $v^{p}(X)=v^{p+1}(X)$. For $x \in v^{p}(X)-v^{p+1}(X)$ implies that

1) Cf. J. Leray: Valeurs propres et vecteurs propres d'un endomorphisme completement continu d'un espace vectoriel à voisinages convexes, Acta Scient Math., 12 B (1950), which will be referred to as [L].

2) When I have almost finished the present note, Professor M. Nagumo called my attension to M. Altman's results: M. Altman: On linear functional equations in locally convex linear topological spaces, Studia Math., 13 (1953). 
for some $f \in X^{*} f(x) \neq 0$ and $\left.f\left(v^{p+1}\right)(X)\right)=0$ since $v^{\text {o+1 }}(X)$ is closed by $[\mathrm{L}]$, Lemma 6.2 and so $f \in v^{*^{-p-1}}(0)=v^{*^{-p}}(0)$. Thus $f(x)=f\left(v^{p}(y)\right)$ $=v^{*^{\mathrm{p}}} f(y)=0$, which is a contradiction. On the other hand $v(X) \subseteq X$ implies $v^{\rho+1}(X) \subseteq v^{\rho}(X)$ for any integer $\rho$, since $v$ is one-to-one, which is also a contradiction.

Lemma 3. Under the same assumption of Lemma 2, $(t-I)^{-1}$ is continuous.

For, on the contrary, we suppose that there is a directed set $\left\{x_{\alpha}\right\}$ such that $x_{\alpha} \rightarrow 0$ and such that $v^{-1}\left(x_{\alpha}\right)+0$. Then for any $\alpha$ there is an $\alpha^{\prime}$ such that $\alpha<\alpha^{\prime}$ and $v^{-1}\left(x_{\alpha^{\prime}}\right) \notin V$ for a fixed neighbourhood $V$ of 0 , which is contained in $U$. Obviously for some $\lambda_{\alpha^{\prime}}<1$ $v^{-1}\left(\lambda_{\alpha^{\prime}} x_{\alpha^{\prime}}\right) \in$ Boundary of $V$. Putting $y_{\alpha^{\prime}}=v^{-1}\left(\lambda_{\alpha^{\prime}} x_{\alpha^{\prime}}\right), y_{\alpha^{\prime}} \in$ Boundary of $V$ and $v\left(y_{\alpha^{\prime}}\right) \rightarrow 0$. Then since $t\left(y_{\alpha^{\prime}}\right)$ is contained in $\overline{t(U)}$, there is an element $u_{0}$ and directed set $\left\{t\left(z_{\beta}\right)\right\}$ such that $\left\{t\left(z_{\beta}\right)\right\}$ is contained in $\left\{t\left(y_{\alpha^{\prime}}\right)\right\}$ and such that $v\left(z_{\beta}\right) \rightarrow 0$ and $t\left(z_{\beta}\right) \rightarrow u_{0}$. Since $v\left(z_{\beta}\right)=t\left(z_{\beta}\right)-z_{\beta}$, $z_{\beta} \rightarrow u_{0}$ and so $t\left(z_{\beta}\right) \rightarrow t\left(u_{0}\right)$. Thus we have $t\left(u_{0}\right)=u_{0}$ and $u_{0} \in$ Boundary of $V$ hence $u_{0} \neq 0$. This is a contradiction.

By the above lemmas we have the following

Theorem 1. Let $t$ be a completely continuous operator without eigenvalue 1. Then $t-1$ maps $X$ onto itself and its inverse is continuous.

By Riesz theorem generalized by Leray and by Lemma 1, applying simple algebraic consideration used by Leray we obtain at once the following

Theorem 2. Let $t$ be a completely continuous operator on locally convex vector space. Then for $t$ and its adjoint $t^{*}$ on the dual space with the strong topology and $\tau\left(X^{*}, X\right)$-topology ${ }^{3}$ respectively, the Riesz and Schauder theorems are valid except that $t^{*}$ is completely continuous in those topologies.

For $t^{*}$ is completely continuous in the topology used in Lemma 1, so by $[\mathrm{L}]$, Theorem 11.1, the theorems concerning eigenvalues and eigenvectors are valid. Furthermore the topology in Lemma 1 is weaker than $r\left(X^{*}, X\right)$-topology, hence $\left(t^{*}-\lambda I\right)(X)$ is closed in that topology for any $\lambda \neq 0$ and so $\left(t^{*}-\lambda I\right)\left(X^{*}\right),(t-\bar{\lambda} I)^{-1}(0)$ and $\left(t^{*}-\lambda I\right)^{-1}(0)$, $(t-\bar{\lambda} I)(X)$ are completely orthogonal pairs. Furthermore if $\lambda$ is not an eigenvalue of $t^{*}$, then $\bar{\lambda}$ is not an eigenvalue of $t$. Hence $t-\bar{\lambda} I$ is onto and $(t-\bar{\lambda} I)^{-1}$ is continuous, so $\left(t^{*}-\lambda I\right)^{-1}$ is continuous in our topologies, where $\bar{t}$ is the conjugate number of $t$.

2. Iterations of operators. As an example shows, it is not always true that for any completely continuous operator

3) Cf. N. Bourbaki: Sur certain espaces vectoriels topologiques, Ann. Inst. Fourier, I (1949). 
$t$, the adjoint $t^{*}$ is completely continuous in the strong topology or in $\tau\left(X^{*}, X\right)$-topology. For instance, , $^{4)}$ let $K(x, y)$ be a measurable and bounded (real valued) function on the unit square $[0,1] \times[0,1]$ such that the operator $t_{1}$ with the kernel $K(x, y)$ on $L^{1}(0,1)$ into itself: $t_{1}(f)(x)=\int f(y) K(x, y) d y$, is not completely continuous. Let $M$ be a space of all bounded measurable functions on $[0,1]$ with the weakest of those topologies which are stronger than the $L^{2}$-topology and the weak topology relative to $L^{1}$. Then the operator $t$ on $M$ with the kernel $\overline{K(y, x)}: t(f)(x)=\int \overline{K(y, x)} f(y) d y$, is completely continuous in our topology. But its conjugate space with the strong topology and with $\tau\left(X^{*}, X\right)$-topology respectively are $L^{1}$ and the adjoint of $t$ is $t_{1}$, which is not completely continuous.

But we have

Theorem 3. Let $t$ be a completely continuous operator on $X$. Then $t^{*} \cdot t^{*}$ is completely continuous operator in the dual space $X^{*}$ with the strong topology and with $z\left(X^{*}, X\right)$-topology respectively.

For, $(t(U))^{0}$ is a neighbourhood of 0 in $X^{*}$ in our topologies and $t^{*}\left((t(U))^{0}\right) \subset U^{0}$, hence we have only to show that $t^{*}\left(U^{0}\right)$ is compact. But it is accomplished by usual methods as in the proof of Lemma 2.

Theorem 4.5) Let $t$ be a continuous operator such that $t^{n}$ is completely continuous for some $n \geqq 1$. Then for $t$ and $t^{*}$ in the dual space with the strong topology, the Riesz and Schauder Theorem is valid except that for any eigenvalue $\lambda,(t-\lambda I)^{-1}(0),\left(t^{*}-\bar{\lambda} I\right)\left(X^{*}\right)$ and $(t-\lambda I)(X),\left(t^{*}-\bar{\lambda} I\right)^{-1}(0)$ are completely orthogonal pairs respectively. Furthermore it may be replaced by $\tau\left(X^{*}, X\right)$-topology instead of the strong one.

For by Theorem 3 , for some $m t^{m}$ and $t^{*^{m}}$ are completely continuous and we easily see that $\lambda$ is an eigenvalue of $t^{m}$ if and only if a value of $\lambda^{\frac{1}{m}}$ is an eigenvalue of $t$. Furthermore $\left(t^{m}-\lambda^{m}\right)^{-1}(0)$ $\supset(t-\lambda)^{-1}(0)$ and for some integer $\rho>0\left(t^{m}-\lambda^{m}\right)^{-p}(0)$ and $\left(t^{*^{m}}-\bar{\lambda}^{m}\right)^{-\mathrm{P}}(0)$ are dual. Moreover $t\left(\left(t^{m}-\lambda^{m}\right)^{-\mathrm{p}}(0)\right) \subset\left(t^{m}-\lambda^{m}\right)^{-\mathrm{p}}(0)$, hence $\operatorname{dim}\left(\left(t-\lambda^{-1}(0)\right)\right.$ $=\operatorname{dim}\left(\left(t^{*}-\bar{\lambda}\right)^{-1}(0)\right)$. Accordingly by a simple consideration we see that the above statement is true.

3. M. Altman call an operation $t$ on $X$ into itself to be completely continuous if for some neighbourhood $U$ of 0 in $X$ any infinite set of $t(U)$ has a limit point in $X$ and give the proof of the

4) Cf. K. Yosida, Y. Mimura, and S. Kakutani: Integral operator with bounded kernel, Proc. Imp. Acad., 14 (1938).

5) Cf. K. Yosida: Normed rings and spectral theorems, II, Proc. Imp. Acad, 19 (1943). 
corresponding Riesz and Schauder Theorem in this case using the Riesz and Schauder Theorem on Banach spaces.

Here we remark that by using M. Altman's simple and useful consideration one see that $t$ and its extension $\bar{t}$ over the completion $\bar{X}$ of $X$ have the same eigenvalues and same eigenvectors. Therefore the main theorems with respect to $t$ follows at once from the corresponding theorems with respect to $\vec{t}$. 